\begin{tabular}{|l|l|}
\hline $\begin{array}{l}\text { Instituto de } \\
\text { Geriatria e Gerontologia }\end{array}$ & $\begin{array}{l}\text { Pan American Journal of Aging Research } \\
\text { PAJAR, Porto Alegre, v. 8, p. 1-9, jan.-dez. } 2020 \\
\text { ISSN-L: 2357-9641 }\end{array}$ \\
\hline http://dx.doi.org/10.15448/2357-9641.2020.1.36528 \\
\cline { 2 - 3 }
\end{tabular}

ARTIGO ORIGINAL

\title{
Avaliação farmacológica de medicamentos usados por idosos frequentadores de uma drogaria privada
}

\author{
Pharmacological evaluation of drugs used by elderly at a private drugstore \\ Evaluación farmacológica de medicamentos utilizados por ancianos en una farmacia \\ privada
}

\section{Grasiela Valer ${ }^{1}$}

orcid.org/0000-0001-5616-0263

grasiela.valer@universo.univates.br

\section{Carla Kauffmann ${ }^{1}$}

orcid.org/0000-0003-4141-7032

carla.kauffmann@univates.br

\section{Marinês Pérsigo Morais \\ Rigo $^{1}$}

orcid.org/0000-0002-9524-6403

mpmr@univates.br

\section{Luísa Scheer Ely}

Martines $^{1}$

orcid.org/0000-0002-1301-3885

luisa.ely@univates.br

Received on: Nov. $27^{\text {th }}, 2019$. Approved on: Mar. $12^{\text {th }}, 2020$ Published on: Sep. 17, 2020

\section{Resumo}

Objetivo: realizar a avaliação da farmacoterapia de idosos frequentadores de uma drogaria privada.

Método: a pesquisa abrangeu um estudo transversal realizado no período de junho a setembro de 2019, em uma drogaria privada, conveniada ao Programa Farmácia Popular do Brasil, localizada no municipio de Nova Bréscia/RS. Para a coleta de dados foi aplicado um questionário, a fim de analisar a farmacoterapia e os hábitos de vida dos idosos participantes da pesquisa na drogaria selecionada. Resultados: a população pesquisada envolveu 81 idosos, sendo a maioria composta por mulheres $(69,1 \%$ ) e a idade média dos participantes foi de 69,4 anos, predominando aqueles na faixa de 60 a 69 anos (53,1\%). A grande maioria dos entrevistados $(66,7 \%)$ se automedica; $36(44,4 \%)$ pessoas foram classificadas com o nivel de polifarmácia maior e uma média de 4,64 medicamentos por pessoas. No total, foram 45 pessoas $(55,6 \%)$ que tiveram algum tipo de interação medicamentosa, variando uma média de 2,72 interações por pessoa. Na pesquisa, foram encontrados 59 medicamentos que são considerados inapropriados para os idosos de acordo com os Critérios de Beers.

Conclusão: o envelhecimento predispõe os idosos a um aumento do uso de medicamentos, aumentando o nível de polifarmácia e as chances de ter interações medicamentosas. Assim, destaca-se a importância do profissional farmacêutico em orientá-los sobre os medicamentos, sobre a não utilização de alguns fármacos, o uso de doses adequadas, acompanhamento da terapia farmacológica, como a sua eficácia e, dessa forma, contribuir para uma qualidade de vida melhor.

Palavras-chave: envelhecimento, idoso, qualidade de vida, prescrições de medicamentos.

\section{Abstract}

Goal: perform an evaluation of the pharmacotherapy of elderly people attending a private drugstore in the city of Nova Brescia/RS.

Method: the research covered a cross-sectional study conducted from June to September 2019, in a private drugstore affiliated to the Popular Pharmacy Program of Brazil, located in Nova Brescia/RS. For data collection, a questionnaire was applied in order to analyze the pharmacotherapy and lifestyle of the senior participants of the research in said drugstore.

Results: the researched population involved 81 people, most of them women (69.1\%). The average age of the participants was 69.4 years old, with predominance in the $60-69$ age group (53.1\%). Most of the respondents (66.7\%) self-medicated; $36(44.4 \%)$ people were classified with high polypharmacy level and an average of 4.64 drugs per person. In total, 45 people (55.6\%) had some kind of drug interaction, varying an average of 2.72 interactions per person. In the research, it was found 59 drugs that are considered inappropriate for the elderly according to the Beers Criterion. Conclusion: aging predispose elderly to increased use of medications, resulting on higher levels of polypharmacy, and the chances of having drug interactions. Thus, the importance of the pharmaceutical professional in guiding them on 
taking medicine, on the non-use of some drugs, the use of appropriate doses, monitoring of pharmacological therapy, as their effectiveness, contributing to a better quality of life.

Keywords: aging, aged, quality of life, drug prescriptions.

\section{Resumen}

Objetivo: realizar una evaluación de la farmacoterapia en ancianos que frequentan una droguería privada en la ciudad de Nova Bréscia/RS.

Método: la investigación incluyó un estudio transversal realizado de junio a septiembre de 2019, en una drogueria privada, afiliada al "Programa Farmácia Popular do Brasil" y ubicada en la ciudad de Nova Bréscia/RS. Para recopilar los datos, se realizó un cuestionario para analizar la farmacoterapia y el estilo de vida de los participantes ancianos de la investigación, que van a la drogueria seleccionada.

Resultados: la población encuestada incluyó a 81 ancianos, la mayoria mujeres $(69.1 \%)$ con edad promedio de los participantes fue de 69.4 años, predominando los de 60 a 69 años (53.1\%). La gran mayoría de los encuestados (66.7\%) se automedica; 36 (44.4\%) personas fueron clasificadas con el nivel más alto de polifarmacia y un promedio de 4.64 drogas por persona. En total, 45 personas (55.6\%) tuvieron algún tipo de interacción farmacológica, variando un promedio de 2.72 interacciones por persona. En la investigación, encontramos 59 medicamentos que se consideran inapropiados para los ancianos según el Criterio de Beers.

Conclusión: envejecimiento predispone los ancianos a un aumento del uso de medicamentos, crecendo los niveles de polifarmacia y las posibilidades de haber interacciones de medicamentos. Así, se destaca la importancia del farmacéutico para informarles acerca de los fármacos, sobre la no-utilización de algunos medicamentos, el uso de dosis apropiadas, el seguimiento de la terapia con medicamentos, como su eficacia, y por lo tanto contribuir para una mejor calidad de vida. Palabras clave: envejecimiento, anciano, calidad de vida, prescripciones de medicamentos.

\section{Introdução}

Sabe-se que os medicamentos são utilizados pela população para tratamento de doenças crônicas e isso pode ser de uso pontual ou contínuo, em diferentes níveis de polifarmácia. A polifarmácia é conceituada pelo uso de dois ou mais medicamentos para um ou vários problemas de saúde. Sua prática pode acarretar reações adversas e interações medicamentosas, principalmente em idosos, que são os mais vulneráveis a esses problemas. ${ }^{1}$

Portanto, a forma como os medicamentos são utilizados interfere na sua ação de forma diferente em cada organismo. Além disso, existem alterações conforme a idade do usuário. ${ }^{2}$ Dessa forma, pessoas idosas não apresentam as mesmas respostas aos medicamentos como as demais, tendo em vista alterações tanto farmacocinéticas, quanto farmacodinâmicas próprias do envelhecimento. ${ }^{3}$

Segundo Lopes et al. (2016)5, a grande maioria dos idosos apresenta várias doenças crônicas ou limitações que necessitam de medicamentos de uso contínuo. Esses medicamentos, apesar de serem utilizados para tratamento de enfermidades, causam riscos à saúde. Por essa razão, existem medicamentos que são classificados como potencialmente inapropriados para idosos, já que os riscos são maiores que os benefícios. Os profissionais da saúde utilizam como referência os Critérios de Beers (2019), que constituem uma lista na qual são identificados os medicamentos potencialmente inapropriados para idosos e seus possiveis riscos para indivíduos dessa faixa etária. ${ }^{6}$

Em relação aos niveis de polifarmácia, a utilização de vários medicamentos pode resultar em interações entre eles, podendo aumentar ou diminuir seus efeitos, causando consequências para o paciente. ${ }^{5}$ Desse modo, para evitar efeitos adversos, que podem resultar em complicações à saúde è̀ qualidade de vida de idosos, é essencial que se preconize o uso racional de medicamentos.?

Com isso, destaca-se o papel do farmacêutico na prática de Atenção Farmacêutica, uma vez que esse profissional é o responsável por interagir com o paciente, objetivando o atendimento às suas necessidades relacionadas aos medicamentos. Assim, busca-se a cura ou a diminuição da doença/sintomas e previne-se que novas doenças se instalem no organismo do individuo. ${ }^{8}$

Em vista do exposto, o presente trabalho objetivou realizar uma avaliação da farmacoterapia de idosos frequentadores de uma drogaria privada.

\section{Métodos}

A pesquisa foi um estudo transversal realizado no período de junho a setembro de 2019, em uma drogaria privada, conveniada ao Programa Farmácia Popular do Brasil, localizada no municipio de Nova Bréscia/RS. O presente estudo teve como critérios de inclusão indivíduos idosos, com idade igual ou superior a 60 anos, frequentadores da farmácia selecionada, que fazem uso de medicamentos do programa Farmácia Popular. Como citério de 
exclusão, foi analisado se o participante possuia condições cognitivas para responder o questionário. Porém, nenhum idoso foi excluído por esse motivo.

Em torno de 200 pessoas utilizavam o programa Farmácia Popular mensalmente, porém dessas, apenas 83 possuiam mais de 60 anos. Para a pesquisa, foram convidados 83 indivíduos, contudo 1 pessoa faleceu nesse período e outra não aceitou responder, totalizando em 81 participantes.

Para a coleta de dados, foi aplicado um questionário com perguntas e respostas abertas e fechadas a fim de analisar a farmacoterapia e os hábitos de vida dos idosos participantes da pesquisa. Essa coleta de dados foi realizada na farmácia, mas, para isso, foi solicitada uma autorização do proprietário, sendo os questionários aplicados na forma de entrevista. Destaca-se que os participantes assinaram o Termo de Consentimento Livre e Esclarecido e que esta pesquisa foi aprovada pelo Comitê de Ética e Pesquisa da Universidade do Vale do Taquari - Univates, sob o número 2.905.211.

Os dados coletados foram tabulados em banco de dados e analisados por meio de frequência, média e desvio padrão no programa Excel ${ }^{\circledR}$. Esses dados possibilitaram a identificação do perfil socioeconômico dos usuários idosos da farmácia, bem como os possiveis problemas relacionados à farmacoterapia dos idosos. Dentre esses problemas, foi possivel identificar a polifarmácia por diferentes pontos de corte, quando varia de 2 a 4 medicamentos ou quando for 5 ou mais medicamentos, catalogar medicamentos potencialmente inapropriados para idosos, de acordo com os Critérios de Beers (2019), verificar possiveis interações medicamentosas, por meio da base de dados UpToDate ${ }^{\circledR}$, e classificar as classes terapêuticas de cada medicamento utilizando o Anatomical Therapeutic Chemical (ATC).

\section{Resultados}

No presente estudo, a população pesquisada envolveu 81 idosos, com idade média de 69,4 \$7,0 anos, variando de 60 a 88 anos, predominando aqueles na faixa de 60 a 69 anos $(53,1 \%)$ e sendo a maioria composta por mulheres $(69,1 \%)$. Em relação à escolaridade, constatou-se que a maioria dos entrevistados (72,8\%) não concluiu o ensino fundamental; enquanto a renda da maioria dos entrevistados (77,8\%) era de R\$998,00 a R\$1.996,00 mensais. Em relação ao estado civil, predominaram indivíduos casados (72,8\%) e viúvos (18,5\%). Por fim, constatou-se que a maioria dos entrevistados residia com algum familiar $(81,5 \%)$, sendo a maioria $(63,6 \%)$ com duas pessoas (Tabela 1 ).

TABELA 1 - Caracterização sociodemográfica da população idosa entrevistada $(n=81)$.

\begin{tabular}{|c|c|c|}
\hline Variável & $\mathbf{N}$ & $\%$ \\
\hline \multicolumn{3}{|l|}{ Sexo } \\
\hline Feminino & 56 & 69,1 \\
\hline Masculino & 25 & 30,9 \\
\hline
\end{tabular}

\section{Faixa etária}

$\begin{array}{lll}60 \text { a } 69 \text { anos } & 43 & 53,1 \\ 70 \text { a } 79 \text { anos } & 31 & 38,3 \\ 80 \text { a } 89 \text { anos } & 7 & 8,6\end{array}$

\section{Escolaridade}

Ensino fundamental incompleto

Ensino médio completo

Ensino superior completo

\section{Estado civil}

$\begin{array}{lcc}\text { Solteiro } & 5 & 6,2 \\ \text { Casado } & 59 & 72,8 \\ \text { Viúvo } & 15 & 18,5 \\ \text { Separado } & 2 & 2,5\end{array}$

\section{Reside}

$\begin{array}{lll}\text { Sozinho } & 15 & 18,5 \\ \text { Com familiar(es) } & 66 & 81,5\end{array}$

\section{Dos que moram com familiares}

$\begin{array}{lcc}\text { Moram com } 2 \text { pessoas } & 42 & 63,6 \\ & & \\ \text { Moram com 3 pessoas } & 20 & 30,3 \\ \text { Moram com 4 pessoas } & 4 & 6,1\end{array}$

\section{Renda}

\begin{tabular}{lcc}
$\mathrm{R} \$ 998,00$ a $\mathrm{R} \$ 1996,00$ & 63 & 77,8 \\
$\mathrm{R} \$ 2800,00$ a $\mathrm{R} \$ 3396,00$ & 9 & 11,1 \\
$\mathrm{R} \$ 4100,00$ a $\mathrm{R} \$ 6800,00$ & 9 & 11,1 \\
\hline TOTAL & 81 & 100
\end{tabular}


Verificou-se que, em relação à ajuda no uso de medicamentos, 70 entrevistados $(86,4 \%)$ não recebiam auxilio, e dos que recebiam, 13,6\%, era referente ao auxilio do cônjuge (54.5\%). Entretanto, 64 entrevistados $(79,0 \%)$ relataram que não esqueceram de tomar os medicamentos no mês anterior ao da realização da pesquisa. Além disso, 75 idosos $(92,6 \%)$ relataram não ter faltas de medicamentos e 53 (65,4\%) não tiveram sobras de medicamentos no decorrer do mês.

A maioria das consultas médicas foram realizadas através da Unidade Básica de Saúde (UBS) ( $n=40 ; 49,4 \%$ ), particular ( $n=28 ; 34,6 \%$ ) ou que frequentavam tanto a UBS quanto clinicas particulares ( $n=13 ; 16,0 \%$ ). Dos entrevistados, $67(82,72 \%)$ referiram ter problemas cardiacos. No entanto, na especialidade, 77 entrevistados $(60,6 \%)$, responderam procurar clínico geral e, em seguida, 19 (15,0\%) individuos declararam procurar cardiologista. Dos participantes da pesquisa, $51(63,0 \%)$ tinham o hábito de verificar a pressão arterial, sendo esse monitoramento realizado em casa por 34 entrevistados (87,2\%). Já o monitoramento da glicemia, foi realizado por 14 (17,3\%) idosos, desses, 7 (50,0\%) realizavam o teste em casa, sendo que $9(11,11 \%)$ relataram ter diabetes.

Dos entrevistados, 20 (24,7\%) consumiam bebida alcoólica e desses, 16 (72,7\%) consumiam vinho.
Em relação às atividades físicas, 41 (50,6\%) dos entrevistados praticavam alguma atividade física e desses, 22 (43,1\%) realizavam caminhadas diárias. Foi questionado sobre a participação em grupos de convivência, bem como as atividades de lazer realizadas pelos idosos; 36 pessoas $(44,4 \%$ ) participavam de grupos de convivência, sendo 22 (51,2\%) no grupo da terceira idade e 20 (24,7\%) realizavam atividades de lazer, sendo desses oito (33.3\%) jogam cartas.

A média de medicamentos utilizados pelos idosos foi de 4,64 medicamentos por pessoa, variando de no mínimo um e no máximo 16 medicamentos. Classificando o nível de polifarmácia, 36 pessoas $(44,4 \%$ ) foram classificadas com o nivel de polifarmácia maior, ou seja, faziam uso de cinco ou mais medicamentos, 35 (43,2\%) pessoas foram classificadas com o nível menor de polifarmácia, pois faziam o uso de dois a quatro medicamentos e 10 (12,3\%) pessoas não faziam uso de polifarmácia, pois utilizavam somente um medicamento.

Em relação às classes de medicamentos utilizadas, a classe No6 foi a mais utilizada (2,7\%), que refere aos psicoanalépticos pertencentes ao grupo do Sistema Nervoso, seguido pela classe Cog, representada pelos fármacos que atuam no sistema renina angiotensina $(2,1 \%)$, pertencentes ao grupo do Sistema Cardiovascular (Tabela 2).

TABELA 2 - Classificação das dez classes de medicamentos mais utilizadas pelos idosos conforme a ATC.

\begin{tabular}{llcc}
\hline \multicolumn{1}{c}{ Classificação } & \multicolumn{1}{c}{ Grupo } & $\begin{array}{c}\mathbf{N}^{\circ} \text { de } \\
\text { medicamentos }\end{array}$ & \% \\
\hline No6 - Psicoanalépticos & Sistema Nervoso & 10 & 2,7 \\
$\begin{array}{l}\text { Co9- Fármacos que atuam no sistema renina } \\
\text { angiotensina }\end{array}$ & Sistema Cardiovascular & 8 & 2,1 \\
A02- Antiácidos, antiulcerosos e anti-inflamatórios & Trato alimentar e metabolismo & 7 & 1,9 \\
C10- Fármacos redutores dos lipídeos & Sistema Cardiovascular & 6 & 1,6 \\
B01-Agentes antitrombóticos & Fármacos que atuam no sistema & 5 & 1,3 \\
C07-Agentes betabloqueadores & hematopoiético & 5 & 1,3 \\
No3- Antiepiléticos & Sistema Cardiovascular & 5 & 1,3 \\
A10- Fármacos usados no diabetes & Sistema Nervoso & 4 \\
A11- Vitaminas & Trato alimentar e metabolismo & 1,1 \\
C03- Diuréticos & Trato alimentar e metabolismo & 4 & 1,1 \\
\hline
\end{tabular}


A Tabela 3 mostra a classificação das 10 interações mais frequentes na pesquisa. No total, foram 45 pessoas $(55,6 \%)$ que tiveram algum tipo de interação medicamentosa, variando uma média de 2,72 interações por pessoa, de no mínimo um e no máximo 31 interações, totalizando 221 interações medicamentosas.

Verificou-se que 16 (7,2\%) interações foram classificadas como risco $\mathrm{B}$, no qual não há ne- cessidade de realizar nenhuma ação. Além disso, constatou-se $13(16,0 \%)$ pessoas com interação de Risco C, o qual exige um monitoramento de terapia. E, na categoria de risco D, encontrou-se $16(7,2 \%)$ pessoas, para as quais é necessário se considerar a modificação da terapia. Importante ressaltar que nessa classificação das 10 interações que mais apareceram, não teve nenhuma interação de "risco X: evite a combinação".

TABELA 3 - As dez interações medicamentosas mais frequentes nos idosos entrevistados, classificadas segundo a base de dados UpToDate ${ }^{\circledR}$.

\begin{tabular}{|c|c|c|c|}
\hline Interações & $N^{\circ}(\%)$ & Classificação & Consequência \\
\hline $\begin{array}{l}\text { levotiroxina + carbonato } \\
\text { de cálcio }\end{array}$ & $8(3,6)$ & $\begin{array}{l}\text { RISCO D: considerar } \\
\text { modificação da terapia }\end{array}$ & $\begin{array}{l}\text { Os sais de cálcio podem diminuir o efeito } \\
\text { terapêutico dos produtos da tireoide. }\end{array}$ \\
\hline $\begin{array}{l}\text { carbonato de cálcio + } \\
\text { hidroclorotiazida }\end{array}$ & $7(3,2)$ & $\begin{array}{l}\text { Risco C: } \\
\text { monitoramento de } \\
\text { terapia }\end{array}$ & $\begin{array}{l}\text { Diuréticos tiazídicos podem diminuir a } \\
\text { excreção dos sais de cálcio. O uso contínuo } \\
\text { pode resultar em alcalose metabólica. }\end{array}$ \\
\hline $\begin{array}{l}\text { ácido acetilsalicílico + } \\
\text { carbonato de cálcio }\end{array}$ & $6(2,7)$ & $\begin{array}{l}\text { Risco B: Nenhuma } \\
\text { ação necessária }\end{array}$ & $\begin{array}{l}\text { Os antiácidos podem diminuir a } \\
\text { concentração sérica de salicilatos. }\end{array}$ \\
\hline $\begin{array}{l}\text { alendronato + carbonato } \\
\text { de cálcio }\end{array}$ & $4(1,8)$ & $\begin{array}{l}\text { RISCO D:considerar } \\
\text { modificação da terapia }\end{array}$ & $\begin{array}{l}\text { Os produtos contendo cátions polivalentes } \\
\text { podem diminuir a concentração sérica de } \\
\text { derivados de bisfosfonato. }\end{array}$ \\
\hline levotiroxina + omeprazol & $4(1,8)$ & $\begin{array}{l}\text { Risco B: Nenhuma } \\
\text { ação necessária }\end{array}$ & $\begin{array}{l}\text { Os inibidores da bomba de prótons } \\
\text { podem diminuir a concentração sérica } \\
\text { dos produtos da tireoide. }\end{array}$ \\
\hline sinvastatina + verapamil & $4(1,8)$ & $\begin{array}{l}\text { RISCO D:considerar } \\
\text { modificação da terapia }\end{array}$ & $\begin{array}{l}\text { O verapamil pode aumentar a } \\
\text { concentração sérica da sinvastatina. }\end{array}$ \\
\hline $\begin{array}{l}\text { atenolol + carbonato de } \\
\text { cálcio }\end{array}$ & $3(1,3)$ & $\begin{array}{l}\text { Risco B: Nenhuma } \\
\text { ação necessária }\end{array}$ & $\begin{array}{l}\text { Os antiácidos podem diminuir a } \\
\text { concentração sérica do atenolol. }\end{array}$ \\
\hline $\begin{array}{l}\text { enalapril + } \\
\text { hidroclorotiazida }\end{array}$ & $3(1,3)$ & $\begin{array}{l}\text { Risco C: } \\
\text { monitoramento de } \\
\text { terapia }\end{array}$ & $\begin{array}{l}\text { Os diuréticos tiazidicos e semelhantes, } \\
\text { podem potencializar o efeito hipotensor } \\
\text { e o efeito nefrotóxico dos inibidores da } \\
\text { enzima conversora de angiotensina. }\end{array}$ \\
\hline gliclazida + metformina & $3(1,3)$ & $\begin{array}{l}\text { Risco C: monitoramento } \\
\text { de terapia }\end{array}$ & $\begin{array}{l}\text { Agentes antidiabéticos podem aumentar } \\
\text { o efeito hipoglicêmio de agentes } \\
\text { associados a hipoglicemia. }\end{array}$ \\
\hline levotiroxina + furosemida & $3(1,3)$ & $\begin{array}{l}\text { Risco B: Nenhuma } \\
\text { ação necessária }\end{array}$ & $\begin{array}{l}\text { A furosemida pode diminuir a ligação às } \\
\text { proteinas dos produtos da tireoide. }\end{array}$ \\
\hline
\end{tabular}

Dos 376 medicamentos utilizados por todos os idosos incluidos na pesquisa, 59 (15.7\%) foram classificados como potencialmente inapropriados para idosos. Tendo uma média de 0,72 medi- camentos potencialmente inapropriados por pessoa, com no mínimo um medicamento por pessoa e no máximo quatro. 
TABELA 4 - Os dez medicamentos mais frequentes identificados no Critério de Beers (2019).

\begin{tabular}{ccc}
\hline Medicamentos & No & $\%$ \\
\hline amitriptilina & 5 & 8,5 \\
glibenclamida & 5 & 8,5 \\
verapamil & 4 & 6,8 \\
ranitidina & 3 & 5,1 \\
cilostazol & 2 & 3,4 \\
clonazepam & 2 & 3,4 \\
doxazosina & 2 & 3,4 \\
espironolactona & 2 & 3,4 \\
ibuprofeno & 2 & 3,4 \\
lorazepam & 2 & 3,4 \\
\hline
\end{tabular}

Já sobre os descartes de medicamentos, 27 indivíduos (33,3\%) levavam na UBS para ter um descarte adequado, e 34 pessoas (42,0\%) nunca realizaram o descarte adequado, descartavam no lixo comum. Em relação ao local da guarda, $52(64,2 \%)$ guardavam na cozinha.

Quando foram questionados sobre a presença de dores, resfriados ou tosse, 54 pessoas $(66,7 \%)$ responderam que se automedicam e, como primeira escolha de tratamento, 43 pessoas (79,6\%) responderam que são os chás. Entretanto, apenas oito pessoas (9,9\%) responderam que já tiveram dúvidas sobre o tratamento e desses, seis $(75,0 \%)$ procuraram o médico para responder suas dúvidas.

\section{Discussão}

Com a pesquisa, observou-se que a maioria eram mulheres que procuravam a farmácia para fazer uso de medicamentos, pessoas com idade entre $60 \mathrm{e}$ 69 anos, com renda inferior a 2 salários mínimos e com ensino fundamental incompleto. A maioria dos estudos envolvendo o uso de medicamentos por idosos relata que as mulheres são as que mais procuram os serviços de saúde e, consequentemente, utilizam um maior número de medicamentos que os homens. Além disso, tendem a viver mais tempo do que os homens, convivendo por maior tempo com doenças crônicas. . $^{9,10,11}$

As doenças crônicas afetam mais os idosos e as pessoas de baixo poder aquisitivo, condições que resultam do menor acesso e uso aos serviços de saúde. Além disso, estudos mostram a relação entre baixa escolaridade e o uso de polifarmácia e de medicamentos potencialmente inapropriados para idosos. A escolaridade encontra-se diretamente associada com o nivel socioeconômico. As pesquisas apontam que o Sistema Único de Saúde (SUS) carece de um esquema de medicamentos mais adequado para idosos. Os hábitos de prescrição para os usuários do SUS costumam ser influenciados pela relação de medicamentos disponiveis gratuitamente. ${ }^{11,12,13}$

A baixa escolaridade é um fator que afeta à adesão terapêutica. ${ }^{14}$ No presente estudo, a maioria não possui o ensino fundamental completo e, para esses, deve existir um maior cuidado sobre as orientações do tratamento. Outro estudo aponta que a escolaridade é um fator importante nos cuidados com a saúde. O baixo nível escolar pode ocasionar problemas de entendimento da doença, assim como leitura e interpretação das informações sobre os medicamentos e, assim, aumentar os riscos de seu uso incorreto. ${ }^{15}$

A maioria dos idosos relatou apresentar problemas cardiacos. Enquanto poucos relataram ter diabetes mellitus (DM). Apesar do DM acometer indivíduos de diferentes idades, ele se destaca nos idosos em decorrência de essa parcela da população apresentar peculiaridades diferentes de outras faixas etárias. ${ }^{16}$

Na UBS, temos como porta de entrada a consulta com o clínico geral, visando, assim, a redução de custos de outros especialistas e, apenas quando necessário, o encaminhamento para o especialista, buscando a melhor opção para cada tipo de necessidade. ${ }^{17} \mathrm{~A}$ hipertensão arterial é frequentemente responsável pelo desenvolvimento de doenças cardiovasculares, tanto as fatais quanto as não fatais. ${ }^{18}$ Além disso, ela também está relacionada com os hábitos alimentares, e com a prática de exercícios físicos.

Como medidas não farmacológicas, é importante destacar que a atividade física é um importante fator para prevenir e controlar essas doenças. No entanto, as atividades devem ser moderadas e sempre monitoradas por um profis- 
sional adequado. ${ }^{19}$ Além da atividade física, o que também está relacionado a doenças cardiacas, são atividades de lazer e sociais, que ajudam na melhora da qualidade de vida do idoso, sendo alternativas não farmacológicas. ${ }^{20}$

Com relação aos medicamentos utilizados pelos idosos, observa-se que o consumo maior foi para os fármacos da classe No6, que refere aos psicoanalépticos. A maioria dos estudos relata que os medicamentos que atuam no sistema cardiovascular são os mais prevalentes em idosos.13.21 Porém, no estudo de Alves e de Ceballos ${ }^{1}$ ), entre os medicamentos de uso crônico mais utilizados, destacam-se os ansiolíticos da classe dos benzodiazepínicos.

Em relação à polifarmácia, identificou-se que $44,4 \%$ faziam uso de 5 ou mais medicamentos. Pesquisas nacionais que utilizaram o recorte etário de 60 anos ou mais detectaram prevalências de polifarmácia de 10,3\% em Cuiabá (MG), ${ }^{22}$ 28,0\% em Goiânia (GO) ${ }^{23}$ e 32,0\% em Florianópolis (SC). ${ }^{24}$ Deve-se ter cuidado ao realizar comparações entre as prevalências de polifarmácia, visto que as diferenças observadas em estudos sobre o uso de medicamentos podem relacionar-se a fatores como o periodo recordatório, o recorte etário, acesso a serviços de saúde, oferta e prescrição de medicamentos nas distintas localidades, bem como o contexto no momento em que o estudo foi realizado, entre outros. A polifarmácia é um fenômeno expressivo, independentemente do local. ${ }^{9}$

A polifarmácia está diretamente ligada ao aumento de interações medicamentosas. As interações medicamentosas são um dos principais riscos do uso de medicamentos. Quando há interferência de um medicamento com medicamento, ou alimento com medicamento, pode haver aumento ou diminuição do efeito dos medicamentos, podendo prejudicar a saúde do usuário. ${ }^{25}$ No presente estudo, a interação medicamentosa entre levotiroxina e carbonato de cálcio foi a mais frequente entre os fármacos. Em estudo similar,essa interação não apareceu, porém de 204 pessoas participantes, 111 (54,4\%) apresentaram algum tipo de interação. Além disso, os autores encontraram um número máximo de 10 interações medicamentosas por pessoa, ocorridas em dois individuos do estudo. ${ }^{25}$

De acordo com os critérios de Beers o medicamento mais utilizado, classificado como medicamento potencialmente inapropriado para os idosos, foi a amitriptilina. Em um estudo que também foi utilizado o critério de Beers, de 78 medicamentos que apareceram nas prescrições, 15 (19,21\%) foram classificados medicamentos potencialmente inapropriados. ${ }^{26}$ Assim, observa-se que entre os estudos, as porcentagens são semelhantes. Esses critérios têm o objetivo de ajudar o prescritor a identificar, corrigir e prevenir prescrição de medicamentos inadequados ${ }^{23}$, portanto, a utilização desses medicamentos na pesquisa, acaba mostrando a falta de conhecimento ou interesse dos prescritores.

Com a pesquisa, observou-se que a maioria dos idosos se automedicam. Essa prática é comum entre as pessoas nessa faixa etária pois, em decorrência do envelhecimento, dores e propensão a doenças aumentam, podendo acarretar em niveis elevados de polifarmácia, efeitos adversos e interações medicamentosas, possibilitando afetar a qualidade de vida. ${ }^{22}$

\section{Conclusão}

A pesquisa possibilitou a identificação do perfil socioeconômico dos usuários idosos da farmácia, além de identificar os níveis de polifarmácia; classificar as classes terapêuticas de cada medicamento; constatar o uso de medicamentos potencialmente inapropriados para idosos e verificar possiveis interações medicamentosas.

Este estudo teve idosos com baixa escolaridade como participantes, que cuidam de sua própria medicação e se automedicam. Além disso, a maioria foi classificada com o nivel maior de polifarmácia, o que aumenta as chances de haver interações medicamentosas.

Assim, conclui-se a importância de um profissional farmacêutico na prática de Atenção Farmacêutica, sendo esse o responsável por interagir com o paciente, objetivando o atendimento às suas necessidades relacionadas aos medicamentos. Diante disso, busca-se a cura ou a diminuição 
da doença/sintomas e a diminuição de interações medicamentosas, evitando-se, assim, reações adversas e intoxicação, além de possibilitar uma maior qualidade de vida aos pacientes idosos.

\section{Referências}

1. Alves N MC, de Ceballos AGDC. Polifarmácia em idosos do programa universidade aberta à terceira idade. J. Health Biol Sci [Internet]. 2018 [Capturado 2019 Mar. 2019];6(4):412-18. Disponivel em: http:// wwW.scielo.br/scielo.php?script=sci_arttext\&pi$\mathrm{d}=$ S0034-89102017000300315\&lng=pt\&nrm=iso. https: $/ /$ doi.org/10.12662/2317-3076jhbs.v6i4.1910.p412-418.2018.

2. Nascimento, RCRM, Álvares J, Guerra Junior AA, Gomes IC, Silveira MR, Costa E A et al. Polifarmácia: uma realidade na atenção primária do Sistema Único de Saúde. Rev. Saúde Pública [Internet]. 2017 [Capturado 15 março 2019]; 51(suppl.2): 1-19. Disponivel em: http:// www.scielo.br/pdf/rsp/v51s2/pt_0034-8910-rsp-S1518-51-s2-87872017051007136.pdf.

3. Cassoni TCJ Corona LP, Romano-Lieber NS, Secoli SR, Duarte YAO, Lebrão ML. Uso de medicamentos potencialmente inapropriados por idosos do Municipio de São Paulo, Brasil: Estudo SABE. Cad. de Saúde Pública. [Internet]. 2014 [Capturado 2019 Nov 03];30:1708-20. Disponivel em: http:// www.scielo.br/pdf/csp/v30n8/0102-311X-csp-30-8-1708. pdf. https://doi.org/10.1590/0102-311X00055613.

4. Yellepeddi V. Farmacocinética. Princípios da terapia farmacológica. In: Whalen, K. et al. Famacologia Ilustrada. 6.ed. Porto Alegre: Artmed, [Internet]. 2016. [Capturado 2019 Fev 03]. Disponivel em: http://srvd.grupoa.com.br/ uploads/imagensExtra/legado/W/WHALEN_Karen/ Farmacologia_Ilustrada_6ed/Lib/Amostra.pdf

5. Lopes LM, Figueiredo TP, Costa SC, Reis AMM. Utilização de medicamentos potencialmente inapropriados por idosos em domicilio. Utilização de medicamentos potencialmente inapropriados por idosos em domicílio. Ciência \& Saúde Coletiva, 2016;21(11):3429-38. https://doi.org/10.1590/1413-812320152111.14302015.

6. American Geriatrics Society 2015 Beers Criteria Update Expert Panel et al. American Geriatrics Society 2019 updated beers criteria for potentially inappropriate medication use in older adults. J. Am. Geriatr. Soc [Internet]. 2015. ICapturado 2019 Maio 24];63(11):2227-46. Disponivel em: https://Www.ncbi.nlm.nih.gov/pubmed/?ter$m=B y \% 20$ the $\% 202019 \% 20$ American\%20Geriatrics\%20 Society\%20Beers\%20Criteria\%C2\%AE\%20Update\%20 Expert\%20Panel\%5BCorporate\%20Author\%5D. https:// doi.org/10.12957/reuerj.2014.7111.

7. Pinto NBF, Vieira LB, Pereira FMV, Reis AMM, Cassiani SHDB. Interações medicamentosas em prescrições de idosos hipertensos: prevalência e significância clínica IDrug interactions in prescriptions for elderly hypertensive patients: prevalence and clinical significance]. Rev Enf UERJ. 2014:22(6):735-41.
8. Pereira LRL, Freitas O. A evolução da Atenção Farmacêutica e a perspectiva para o Brasil. Rev. Bras. Cienc. Farm. [Internet]. 2008 Dec [capturado 2020 Ago 19]; 44(4):601-12. Disponivel em: http:// www.scielo.br/scielo.php?script=sci_arttext\&pi$\mathrm{d}=\mathrm{S} 1516-93322008000400006 \&$ lng $=e n$. https://doi. org/10.1590/S1516-93322008000400006.

9. Marques PP, Assumpção D, Rezende R, Neri AL, Francisco PMSB. Polifarmácia em idosos comunitários: resultados do estudo Fibra. Rev. Bras. Geriatr. Gerontol. [Internet]. 2019 [Capturado 2019 Dez];22(5):e190118. Disponivel em: http://www.rbgg.com.br/edicoes/v22n5/ RBGG\%20V22n5\%20PORT_2019-0118.pdf

10. Sales AS, Sales MGS, Casotti CAC. Perfil farmacoterapêutico e fatores associados à polifarmácia entre idosos de Aiquara, Bahia, em 2014. Epidemiol. Serv. Saúde. [Internet]. 2017 [Capturado 2019 Dez. 25]; 26(1):12132. Disponivel em: https://www.scielosp.org/pdf/ ress/2017.v26n1/121-132/pt. https://doi.org/10.5123/ S1679-49742017000100013.

11. Heather LB, Irribarem AMV., Dâmaso BA. Inadequação do uso de medicamentos entre idosos em Pelotas, RS. Rev Saúde Pública [Internet]. 2017 [Capturado 2019 Dez. 25];51: 1-12. Disponivel em: https://www.redalyc. org/pdf/672/67249591053.pdf

12. Skaar DD, O'Connor HL. Use of the Beers criteria to identify potentially inappropriate drug use by community-dwelling older dental patients. Oral Surg Oral Med Oral Pathol Oral Radiol. [Internet]. 2017 [Capturado 2019 Dez. 25]; 113(6):714-21. Disponivel em: https://jada.ada. org/article/S0002-8177(17)30077-6/pdf. https://doi. org/10.1016/j.0000.2011.12.009.

13. Santos TRA., Lima DM, Nakatani AYK, Pereira LV, Leal GS, Amaral RG. Consumo de medicamentos por idosos, Goiânia, Brasil. R Saúde Pública [Internet]. 2013 [Capturado 2019 Dez. 25]; 47(1):94-103. Disponivel em: http://www.scielo.br/pdf/rsp/v47n1/13.pdf. https:// doi.org/10.1590/S0034-89102013000100013.

14. Tavares NUL, Bertoldi AD, Mengue SS, Arrais PSD, Luiza VL, Oliveira MA et al. Fatores associados à baixa adesão ao tratamento farmacológico de doenças crônicas no Brasil. Rev. Saúde Pública [Internet]. 2016 [capturado Dez. 25];50( Suppl 2 ):1-10s. Disponível em: http://www.scielo.br/scielo.php?script=sci_arttext\&pid=S0034-89102016000300307\&lng=en. https://doi. org/10.1590/s1518-8787.2016050006150.

15. Loff J. Adesão Ao Tratamento Farmacológico E Não Farmacológico Dos Hipertensos de um Município do Vale do Caí - RS [Trabalho de Conclusão de Curso na Internet]. Novo Hamburgo: Universidade Feevale; 2017. [Capturado 2019 Out. 04]. Disponivel em: https:// biblioteca.feevale.br/Vinculo2/000013/00001336.pdf

16. da Silva LHA. Veja orientações da diretriz para tratamento de diabetes em idosos. Portal Pebmed. [Internet]. 2019. [Capturado 2019 Out. 15]. Disponivel em: https://pebmed.com.br/veja-orientacoes-da-diretriz-para-tratamento-de-diabetes-em-idosos/

17. da Silva CB, Flores CR, Riss LA. Saúde mental de médicos clínicos gerais em unidades básicas de Saúde. Rev. Psicol. Foco [Internet]. 2017 Jul [2019 Out. 10]; 9(13):2-21. Disponivel em: http://revistas.fw.uri.br/index. $\mathrm{php/psicologiaemfoco/article/view/2940/2479}$ 
18. Carvalho MD, Bernal RTI, Andrade SSCA, da Silva MMA, Velasquez-Melendez G. Prevalência e fatores associados com hipertensão arterial autorreferida em adultos brasileiros. Rev. Saúde Pública [Internet]. 2017 [Capturado 2019 Out. 10]:51 Suppl 1:1-11s. Disponivel em: http://www.scielo.br/scielo.php?script=sci_ arttext\&pid $=$ So034-89102017000200313\&lng=en. Epub June 01, 2017. https://doi.org/10.1590/s15188787.2017051000006. https://doi.org/10.1590/s15188787.2017051000006.

19. Acencio FR, Gomes JB, Antunes MD, Bueno GR, Pavanelli GC. Influência do Exercício Físico na Qualidade de Vida de Idosas de um Grupo Social de Tapejara, PR. Rev. Univ. Vale Rio Verde [Internet]. 2017 [Capturado 2019 Out. 12]; 15(2):658-66. Disponivel em: http://periodicos.unincor.br/index.php/revistaunincor/article/view/3893/ pdf_728. https://doi.org/10.5892/ruvrd.v15i2.3893.

20. Oliveira, F. A., Pirajá, W. C., Silva, A. P., \& Primo, C. P. F. Benefícios da prática de atividade física sistematizada no lazer de idosos: algumas considerações. Licere [Internet]. 2015 [Capturado 2019 Out. 12];18(2):262304. Disponivel em: https://www.researchgate.net/ profile/flavio_oliveirag/ publication/ 283641423_beneficios_da_pratica_de_atividade_fisica_sistematizada_no_lazer_de_idosos_algumas_consideracoes_benefits_of_systematised_physical_activity_practice_on_ the_elderly_people's_leasure_some_oconsiderations/ links/5641f1c608aeacfd8937db10.pdf. https://doi. org/10.35699/1981-3171.2015.1110.

21. Martins GA, Acursio FA, Franceschini SCC, Priore SE, Ribeiro AQ. Uso de medicamentos potencialmente inadequados entre idosos do Municipio de Viçosa, Minas Gerais, Brasil: um inquérito de base populacional. Cad. Saúde Pública [Internet]. 2015 [Capturado 2019 Dez. 25l:31(11):1-14. https://WwW.scielosp.org/scielo. php?pid=S0102-311X2015001302401\&script=sci_arttext\&tlng=pt\#. https://doi.org/10.1590/0102-311X00128214.

22. de Almeida NA, Reiners AAO, Azevedo RCS, da Silva AMC, Cardoso JDC, de Souza LC. Prevalência e fatores associados à polifarmácia entre os idosos residentes na comunidade. Rev. Bras. Geriatr. Gerontol. [Internet]. 2017 [Capturado 2019 Out. 16]; 20(1):143-53. Disponivel em: https://www.redalyc.org/pdf/4038/403850707015.pdf

23. Silveira E.A., Dalastra L., Pagotto V. Polifarmácia, doenças crônicas e marcadores nutricionais em idosos. Rev Bras Epidemiol. [Internet]. 2014 [Capturado 2019 Dez 25];17(4):818-29. Disponivel em: https://www.researchgate.net/profile/Erika_Silveira/publication/267927889_Polypharmacy_chronic_diseases_and_nutritional_markers_in_community-dwelling_older_Polifarmacia_doencas_cronicas_e_marcadores_nutricionais_em_idosos/ links/545d7ef20cf295b5615e6d58/Polypharmacy-chronic-diseases-and-nutritional-markers-in-community-dwelling-older-Polifarmacia-doencas-cronicas-e-marcadores-nutricionais-em-idosos.pdf. https://doi. org/10.1590/1809-4503201400040002.

24. Pereira K.G. Peres MA, lop D, Boing AC, Boing A $F$, Aziz $M$ et al. Polifarmácia em idosos: um estudo de base populacional. Rev Bras Epidemiol. [Internet]. 2017 [Capturado 2019 Dez. 25];20(2):335-44. Disponivel em: https://www.scielosp.org/article/rbepid/2017. v2on2/335-344/en/. https://doi.org/10.1590/19805497201700020013 .
25. Garske CCD, de Assis MP, Schneider APH, Machado EO, Morsch LM. Interações medicamentosas potenciais na farmacoterapia de idosos atendidos em farmácia básica do sul do Brasil. Saúde (Santa Maria) [Internet]. 2016 [Capturado 2019 Out. 15];42(2):97-105. Disponivel em: https://periodicos.ufsm.

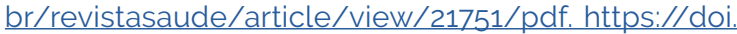
org/10.5902/2236583421751.

26. dos Santos Ferreira, W, da Silva Sobral, MP, Torres VM. Perfil de prescrições atendidas em uma farmácia. Rev Bras Educ Saúde [Internet]. 2019 [Capturado 2019 Out. 22];9(3):100-05. Disponivel em: https://editoraverde.org/gvaa.com.br/revista/index.php/REBES/ article/view/6627/6354

\section{Endereço para correspondência}

Luisa Scheer Ely Martines

Universidade do Vale do Taquari

Av. Avelino Talini, 171

Universitário, CEP 95914014

Lajeado, RS, Brasil 